\title{
Nimodipine Reverses the Elevation of Synaptic Striatal Dopamine and Serotonin During In Vivo Hypoxia
}

\author{
Michael Haile*, ${ }^{*}$, Patricia Broderick ${ }^{2}$, Yong-Sheng Li $^{3}$, Thomas Blanck ${ }^{1}$, David Quartermain ${ }^{3}$ and \\ Alex Bekker ${ }^{1}$ \\ ${ }^{I}$ New York University Langone Medical Center, Department of Anesthesiology, USA \\ ${ }^{2}$ Sophie Davis School of Biomedical Education CUNY, Department of Physiology \& Pharmacology; New York Univer- \\ sity Langone Medical Center, Department of Neurology, USA \\ ${ }^{3}$ New York University Langone Medical Center, Department of Neurology, USA
}

\begin{abstract}
Introduction: Moderate hypoxia has been implicated in the development of delirium. One mechanism may be an increase of Dopamine (DA) and Serotonin (5-HT) levels. Our previous studies indicated that hypoxia increased levels of both neurotransmitters (NT) and that nimodipine (NIMO) administered immediately after hypoxia preserved short term memory. We tested the hypothesis that these observations may be related to a NIMO dependent reduction of hypoxia induced NT elevation.

Methods: Following IACUC approval, In Vivo microvoltammetry sensors (BRODERICK PROBE ${ }^{\circledR}$ ) were implanted in the dorsal striatum of Na Pentobarbital anesthetized adult Sprague-Dawley rats. Extracellular NT levels were recorded for $15 \mathrm{~m}$ during the establishment of baseline values in room air $(\mathrm{N}=6)$. Three sequential thirty-minute periods under hypoxia $(10 \% \mathrm{O} 2)$ followed. First: under hypoxia alone; second after i.p. injection of NIMO $(0.1 \mathrm{mg} / \mathrm{kg})$; and third following i.p. injection of NIMO $(1.0 \mathrm{mg} / \mathrm{kg})$. Measurements were analyzed with ANOVA with post hoc Tukeys test. P-values less than 0.05 were considered significant.
\end{abstract}

Results: NT levels are expressed as percentages of baseline values. Treatment values were averaged over each sequential thirty-minute period following each intervention. Moderate hypoxia resulted in the increase of DA to 172\% (SEM=18) and 5 -HT to $68 \%(\mathrm{SEM}=4)$ above baseline. Under continuing hypoxia, NIMO $(0.1 \mathrm{mg} / \mathrm{kg})$ caused DA levels to fall to $112 \%(\mathrm{SEM}=14)$ and $5-\mathrm{HT}$ to $13 \%(\mathrm{SEM}=5)$ above baseline. NIMO $(1.0 \mathrm{mg} / \mathrm{kg})$ caused DA levels to fall to $34 \%$ $(\mathrm{SEM}=6)$ above baseline and 5-HT to $20 \%(\mathrm{SEM}=2)$ below baseline.

Conclusion: Moderate hypoxia increased levels of DA and 5-HT in the striatum of rats. NIMO administration during ongoing hypoxia caused the levels of both neurotransmitters to fall towards baseline. The results may have implications for understanding and treating cognitive decline due to hypoxia.

Keywords: Moderate hypoxia, neurotransmitters, dopamine, serotonin, L-type, calcium channel blockers, nimodipine.

\section{INTRODUCTION}

Changes in concentrations of the neurotransmitters (NT) dopamine (DA), serotonin (5-HT) and acetylcholine (Ach) [1-3] are associated withhypoxia and implicated in the development of delirium [4]. Delirium is an acute state of confusion that can be brought about by conditions such as hypoxia. It is manifested by temporary cognitive dysfunction, including that of working memory. Hypoxia has also been shown to increase cytosolic levels of calcium [5-7]. Intracellular calcium affects the synthesis and release of several NT [8]. We hypothesized that the relationship between moderate hypoxia and delirium could be related to calcium homeostasis. The role of hypoxia upon NT changes and the role of calcium homeostasis upon NT changes during hypoxia and

*Address correspondence to this author at the NYU Langone Medical Center, Department of Anesthesiology, 550 First Avenue, RR-605, New York, NY 10016, USA; Tel: 212-263-5072; E-mail: hailem01@nyumc.org normoxia with and without nimodipine (NIMO) treatment were examined.

Previous studies showed that moderate hypoxia increased DA and 5-HT in rat striatum [9] and that the L-type calcium channel (LTCC) blocker, NIMO, which crosses the blood brain barrier, prevented working short term memory cognitive defects in mice when administered immediately after moderate hypoxia [10]. Herein, we focused on the synaptic concentrations of DA and 5-HT and extended our previous in vivo hypoxia study to examination of calcium mechanisms.

Neurotransmitter synthesis and release are highly sensitive to hypoxia. DA [11] and 5-HT synthesis [14] have been shown to be hypoxia-dependent. We recorded in vivo synaptic NT concentrations using BRODERICK PROBE ${ }^{\circledR}$ laurate neuromolecular imaging (NMI) biosensors based on microvoltammetry. NMI, allows for NTs to be detected electrochemically within seconds recording actual synaptic concen- 
trations. The amount of NT is directly correlated with the concentrations present [12]

In these studies, we tested our hypothesis that links between hypoxia, NT, and NIMO may be related to an ion channel dependent attenuation of the hypoxia-induced dysregulation of calcium homeostasis. A decrease in intracellular concentrations of calcium during hypoxia may preserve NT homeostasis.

\section{METHODS}

\section{Animals}

After Institutional Animal Care and Use Committee approval fifteen adult male rats (Charles River Laboratories, Raleigh, NC) weighing from 300-340g were fed Purina Rat Chow and water ad libitum on a 12-h dark-light cycle.

\section{Study Design}

Subjects were divided into three groups and neurochemical recordings were conducted every $5 \mathrm{~m}$. Baseline values were established in room air for $15 \mathrm{~m}$ before treatment for all subjects.

For $\mathrm{N}=6$;

1. $30 \mathrm{~m}$ under hypoxia $(10 \% \mathrm{O} 2)$.

2. $30 \mathrm{~m}$ under hypoxia after intraperitoneal (i.p.) injection of NIMO $(0.1 \mathrm{mg} / \mathrm{kg})$.

$3.30 \mathrm{~m}$ under hypoxia after NIMO $(1.0 \mathrm{mg} / \mathrm{kg})$.

For $\mathrm{N}=4$;

$1.90 \mathrm{~m}$ (three $30 \mathrm{~m}$ periods) under hypoxia alone.

For $\mathrm{N}=6$;

$1.30 \mathrm{~m}$ in room air after NIMO $(0.1 \mathrm{mg} / \mathrm{kg})$.

$2.30 \mathrm{~m}$ in room air after NIMO $(1.0 \mathrm{mg} / \mathrm{kg})$.

\section{Procedure}

The protocol closely followed that of Broderick and Gibson [9]. Subjects received i.p. $50 \mathrm{mg} / \mathrm{kg}$ of Pentobarbital $\mathrm{Na}$ in a $6 \%$ solution of distilled water. Temperature was monitored rectally (Fisher Scientific, Fadem, NJ) and maintained at $37.5^{\circ} \mathrm{C} \pm 1.0^{\circ} \mathrm{C}$ with an Aquamatic $\mathrm{K}$ heating pad (Amer. Hosp. Supply, Edison, NJ). A Nonin model 8600V pulse oximeter (Nonin Medical, Plymouth, MN) applied provided oxygen saturation (SpO2). A rat anesthesia mask Kopf \#906A (Kopf Instruments, Tujunga, CA) was used on the snout with the detached thumb portion of a latex surgical glove to make a loose gas seal. An Elite \#799 air pump (Hagen, Montreal, Canada) delivered air during normoxia. $10 \%$ oxygen $/ 90 \%$ nitrogen tanks premixed to $+/-0.1 \%$ of the smallest gas constituent (TW Smith Corp, Brooklyn, NY) were used during hypoxia. Flow rates were $1.5 \mathrm{~L} / \mathrm{m}$ precluding leakage of gases. NIMO (Sigma-Aldrich, St Louis, MO) was dissolved in $60 \%$ polyethylene glycol $/ 40 \%$ methanol by mass then diluted 1:10 with distilled water.

\section{Biosensors}

BRODERICK PROBE ${ }^{\circledR}$ biosensors were manufactured on site. Details of the design and construction of the sensors are published $[13,14]$. Biosensors are pre-calibrated in vitro in a freshly made deoxygenated physiological saline- phosphate buffer solution $(0.01 \mathrm{M}, \mathrm{pH}=7.4$ containing nmol aliquots of DA and 5-HT (99\% purity, Sigma-Aldrich, St. Louis, MO)5).

\section{Surgery}

Pinnal, corneal and leg flexion responses were monitored and supplemental doses of $\mathrm{Na}$ pentobarbital were administered as needed. A stereotactic device (Kopf Stereotaxic, Tujunga, CA) was used. After incision three holes were drilled in the skull. The BRODERICK PROBE ${ }^{\circledR}$ indicator sensor was inserted over a period of $20 \mathrm{~m}$ into the dorsal striatum at stereotaxic coordinates $(\mathrm{AP}=+2.5, \mathrm{ML}=+2.6$, $\mathrm{DV}=-4.0)$ [15] (An $\mathrm{Ag} / \mathrm{AgCl}$ reference electrode was placed in contact with dura, $7 \mathrm{~mm}$ anterior and contralateral to the indicator microelectrode. A stainless steel microauxiliary electrode was placed in contact with the cortex to complete the circuit and provide a ground creating a semiderivative microvoltammetric circuit. Electrodes were connected through a potentiostat. A potential was applied across the sensors to oxidize or reduce the NT in extracellular fluid (ECF). The current resulting from the redox processes in the ECF is plotted as a function of potential in order to create a voltammogram (Fig. 1). Subjects were euthanized by injection of $\mathrm{Na}$ Pentobarbital (100 mg/kg, i.p).

\section{Recording}

Potentials were applied with a CV37 detector (BAS, West Lafayette, IN) from $-0.2 \mathrm{~V}$ to $+0.9 \mathrm{~V}$ with respect to an $\mathrm{Ag} / \mathrm{AgCl}(1 \mathrm{M} \mathrm{NaCl})$ electrode, at a scan rate of $10 \mathrm{mV} / \mathrm{s}$ at time constants of 5 and $1 \mathrm{~s}$ tau. After charging and reduction of neurochemicals in the surrounding tissues, the sensors detected returning oxidation currents. Each scan was completed in 60s. Nonfaradaic charging current was eliminated in the first 25s. DA and 5-HT were detected sequentially in two waveforms with a specific signature determined by its characteristic oxidative potential (voltage). Oxidation potentials for these biosensors, in vivo, are, in the order of detection from lowest to highest; DA [0.14 V] and 5-HT [0.31 V]. Each specified oxidation potential is within a range of $+/$ $0.015 \mathrm{~V}$.

\section{Statistics}

The current that is produced by a specific redox reaction is proportional to the concentration of neurochemicals according to the Cottrell equation: ( $i=$ nFA$\operatorname{coO}(\mathrm{DO}) 1 / 2 / \pi 1 / 2 \mathrm{t} 1 / 2)$. Changes in concentrations of DA and 5 -HT are presented as percent changes to minimize normal between-animal variations such that each subject served as its own control. Measurements were analyzed with ANOVA with post hoc Tukey's test. P-values $<0.05$ were considered significant.

\section{RESULTS}

DA and 5-HT levels are presented as percent changes (percent of control) from baseline to minimize normal between-animal variations. In vivo recordings were completed over 60 s and repeated every 5 minutes. Fig. (1) is a series of recordings from one subject. First is the baseline / control period; second is hypoxia; third is NIMO $(0.1 \mathrm{mg} / \mathrm{kg})$; and fourth is NIMO $(1.0 \mathrm{mg} / \mathrm{kg})$. DA and 5-HT are detected according to their respective oxidative potentials (voltage on the $\mathrm{X}$ axis). Currents recorded were in the order of magni- 


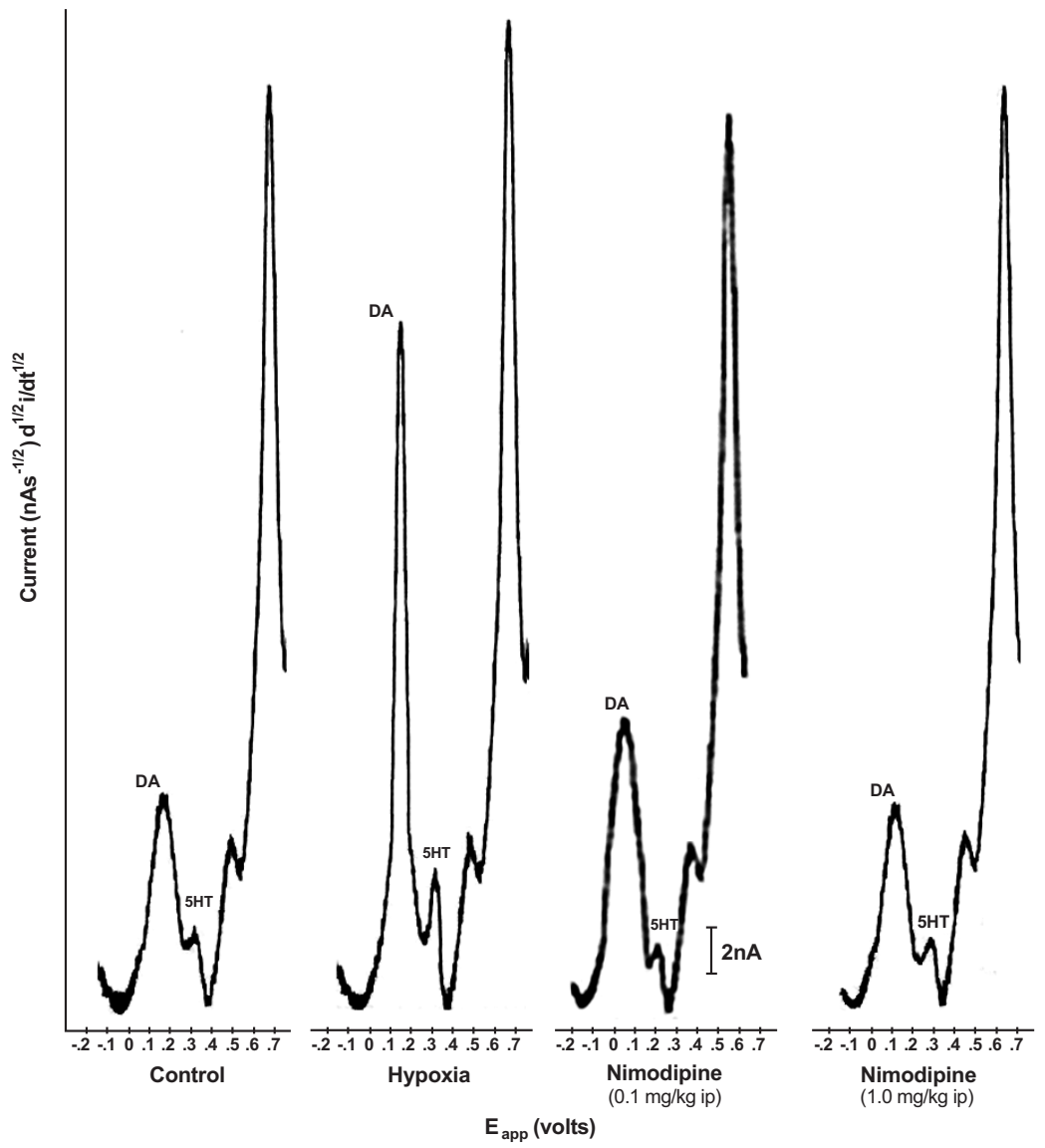

Fig. (1). A representative series of neurotransmitter recordings from dorsal striatum derived from data from six subjects. These scans are a series of recordings from one subject over all four phases of the study. The first recording is from the baseline/control period, the second from the hypoxic period, the third from the first NIMO $(0.1 \mathrm{mg} / \mathrm{kg})$ treatment period, and the fourth from the final NIMO $(1.0 \mathrm{mg} / \mathrm{kg})$ treatment period.

tude of nA (Y axis). According to the Cottrell equation: $(i=$ $\mathrm{nFAcoO}(\mathrm{DO}) 1 / 2 / \pi 1 / 2 \mathrm{t} 1 / 2)$, the current that is produced by a specific redox reaction is proportional to concentration.

For group one, Fig. (2) shows sequential NT concentrations derived from a series of recordings for $\mathrm{N}=6$ rats. First, normoxia $(21 \% \mathrm{O} 2)$, second moderate hypoxia $(10 \% \mathrm{O} 2)$, and third nimodipine $(0.1 \& 1.0 \mathrm{mg} / \mathrm{kg}$ i.p. $)$ are shown. Electrochemical signals are expressed as percentages of baseline values $(100 \%)$ obtained over $15 \mathrm{~m}$. Treatment values were averaged over each sequential $30 \mathrm{~m}$ period after intervention. Hypoxia caused an increase of DA to $172 \%(\mathrm{SEM}=18)$ and 5-HT to $68 \%(\mathrm{SEM}=4)$ above baseline. Under continuing hypoxia, NIMO $(0.1 \mathrm{mg} / \mathrm{kg})$ caused DA concentrations to fall to $112 \%(\mathrm{SEM}=14)$ and 5 -HT to $13 \%(\mathrm{SEM}=5)$ above baseline. Under continuing hypoxia, NIMO $(1.0 \mathrm{mg} / \mathrm{kg})$ caused the DA signal to fall to $34 \%$ (SEM=6) above baseline and the 5-HT signal to fall to $20 \%(\mathrm{SEM}=2)$ below baseline. Hypoxia caused an increase in the striatal concentration of DA and 5-HT.

NIMO $(0.1 \mathrm{mg} / \mathrm{kg})$ decreased concentrations of DA and caused hypoxic-induced concentrations of 5-HT to fall below baseline. NIMO $(1.0 \mathrm{mg} / \mathrm{kg})$ caused the DA signal to fall towards baseline and the 5-HT signal to fall below baseline. Hypoxia caused a decrease of $\mathrm{SpO} 2$ from a baseline of $90.9 \%$ (SEM 0.9) to a hypoxic level of 50.8\% (SEM 1.0). NIMO treatment did not significantly change SpO2. Tem- perature remained stable throughout the study period at 36.6 degrees (SEM 0.1) Centigrade.

For group two, the effect of ongoing hypoxia on striatal NT was observed. Fig. (3) shows sequential NT concentrations derived from a series of recordings in $\mathrm{N}=4$ rats. First, normoxia and then hypoxia are shown. In the first $30 \mathrm{~m}$ period of hypoxia DA increased to $66 \%(\mathrm{SEM}=8)$ and 5 -HT to $37 \%(\mathrm{SEM}=2)$ above baseline. In the second $30 \mathrm{~m}$ period under continuing hypoxia DA concentrations rose to $111 \%$ $(\mathrm{SEM}=8)$ and $5-\mathrm{HT}$ to $52 \%(\mathrm{SEM}=7)$ above baseline. In the third $30 \mathrm{~m}$ period under continuing hypoxia DA concentrations rose to $237 \%(\mathrm{SEM}=29)$ and $5-\mathrm{HT}$ to $145 \%(\mathrm{SEM}=30)$ above baseline. In a final $10 \mathrm{~m}$ period DA concentrations rose to $482 \%$ (SEM=47 and 5-HT to $366 \%$ above baseline (SEM=42). Continuing hypoxia without NIMO treatment resulted in a continual increase in the concentrations of NT. Hypoxia caused a decrease of $\mathrm{SpO} 2$ from baseline to a hypoxic level of $55.6 \%$ (SEM 1.3) over the remainder of the study period. Temperature remained stable throughout the study period at 36.5 degrees (SEM 0.04) Centigrade.

For group three NIMO injection in normoxic rats was evaluated to observe the effect of NIMO upon NT. Fig. (4) shows sequential NT concentrations derived from a series of recordings in $\mathrm{N}=6$ rats. First, normoxia and then NIMO $(0.1$ then $1.0 \mathrm{mg} / \mathrm{kg}$ i.p.) are shown. Normoxic injection of NIMO $(0.1 \mathrm{mg} / \mathrm{kg})$ resulted in the decrease of DA to $40 \%(\mathrm{SEM}=3)$ 


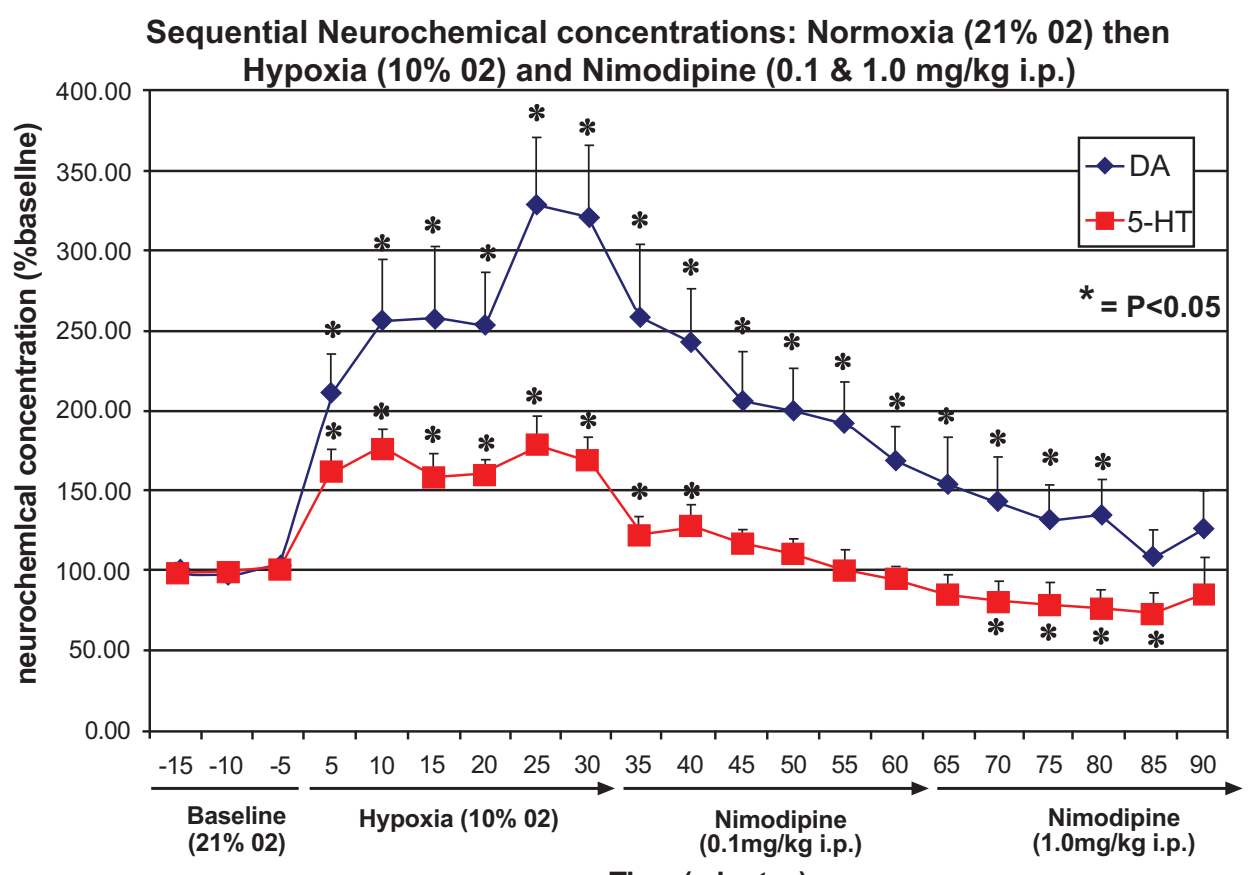

Time (minutes)

Fig. (2). Sequential Neurochemical concentrations: Normoxia $(21 \%$ O2) then Hypoxia $(10 \%$ O2) and Nimodipine $(0.1$ \& $1.0 \mathrm{mg} / \mathrm{kg}$ i.p. $)$. NMI signals for each neurochemical are expressed as percentages of baseline values (100\%) obtained over fifteen minutes. Treatment values were averaged over each sequential thirty minute period following each intervention. Moderate hypoxia resulted in the increase of DA to $172 \%(\mathrm{SEM}=18)$ and 5 -HT to $68 \%(\mathrm{SEM}=4)$ above baseline. Under continuing hypoxia, NIMO (0.1mg/kg) caused DA to fall to $112 \%$ $(\mathrm{SEM}=14)$ and $5-\mathrm{HT}$ to $13 \%(\mathrm{SEM}=5)$ above baseline. Under continuing hypoxia, NIMO (1.0mg/kg) caused DA to fall to $34 \%$ (SEM=6) above baseline and 5-HT to 20\% (SEM=2) below baseline. Measurements were analyzed with ANOVA with post hoc Tukeys test. P-values less than 0.05 were considered significant.

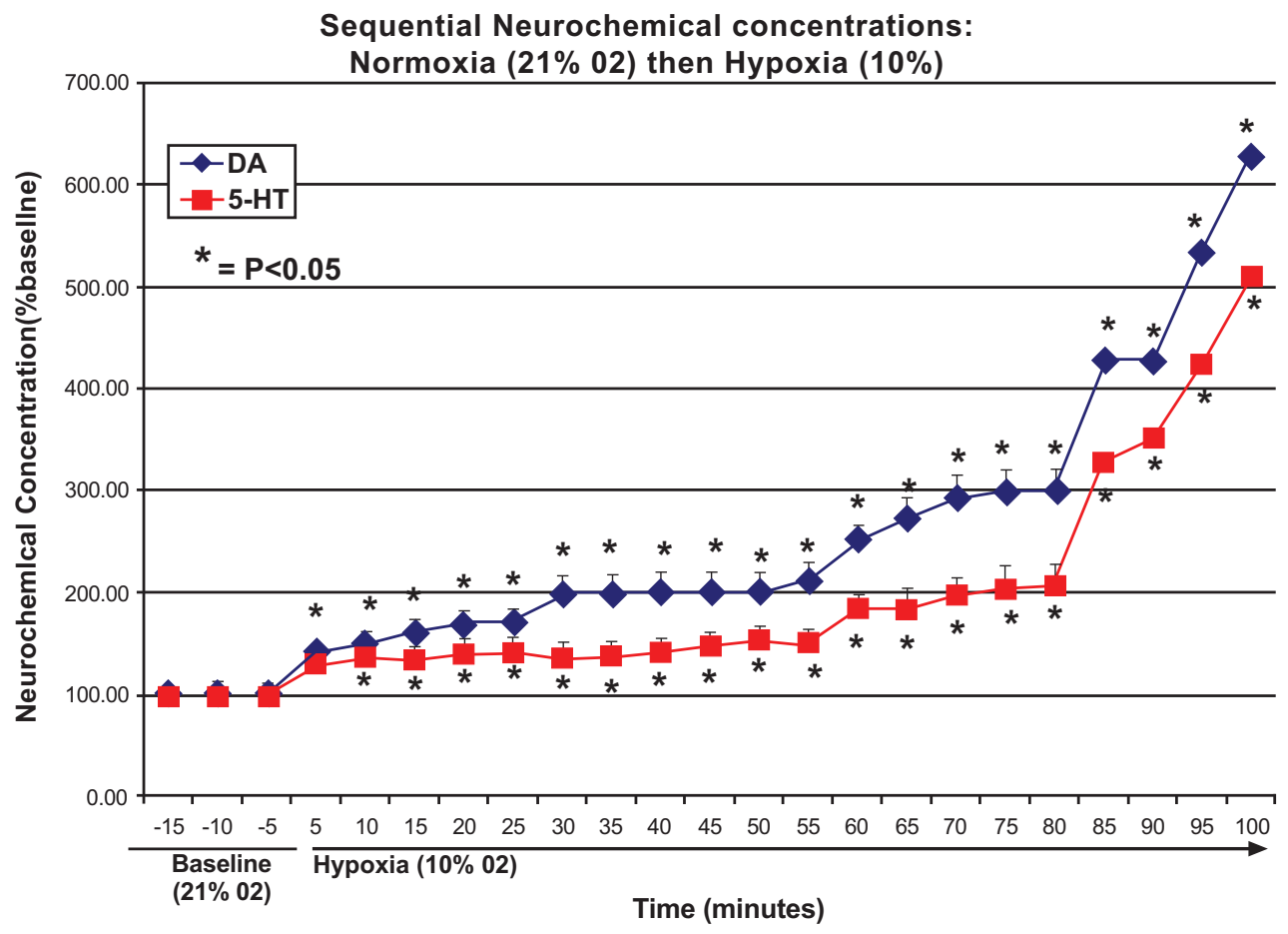

Fig. (3). Sequential Neurochemical concentrations: Normoxia (21\% O2) then Hypoxia (10\%). NMI signals for each neurochemical are expressed as percentages of baseline values (100\%) obtained over fifteen minutes. Treatment values were averaged over each sequential thirty minute period after hypoxia. In the first thirty minute period moderate hypoxia resulted in the increase of DA to 66\% (SEM=8) and 5-HT to $37 \%(\mathrm{SEM}=2)$ above baseline. In the second thirty minute period under continuing hypoxia, DA rose to $111 \%(\mathrm{SEM}=8)$ and $5-\mathrm{HT}$ to $52 \%$ $(\mathrm{SEM}=7)$ above baseline. In the third thirty minute period under continuing hypoxia DA concentrations increased to $237 \%$ (SEM=29) above baseline and 5-HT to $145 \%(\mathrm{SEM}=30)$ above baseline. Measurements were analyzed with ANOVA with post hoc Tukeys test. P-values less than 0.05 were considered significant. 


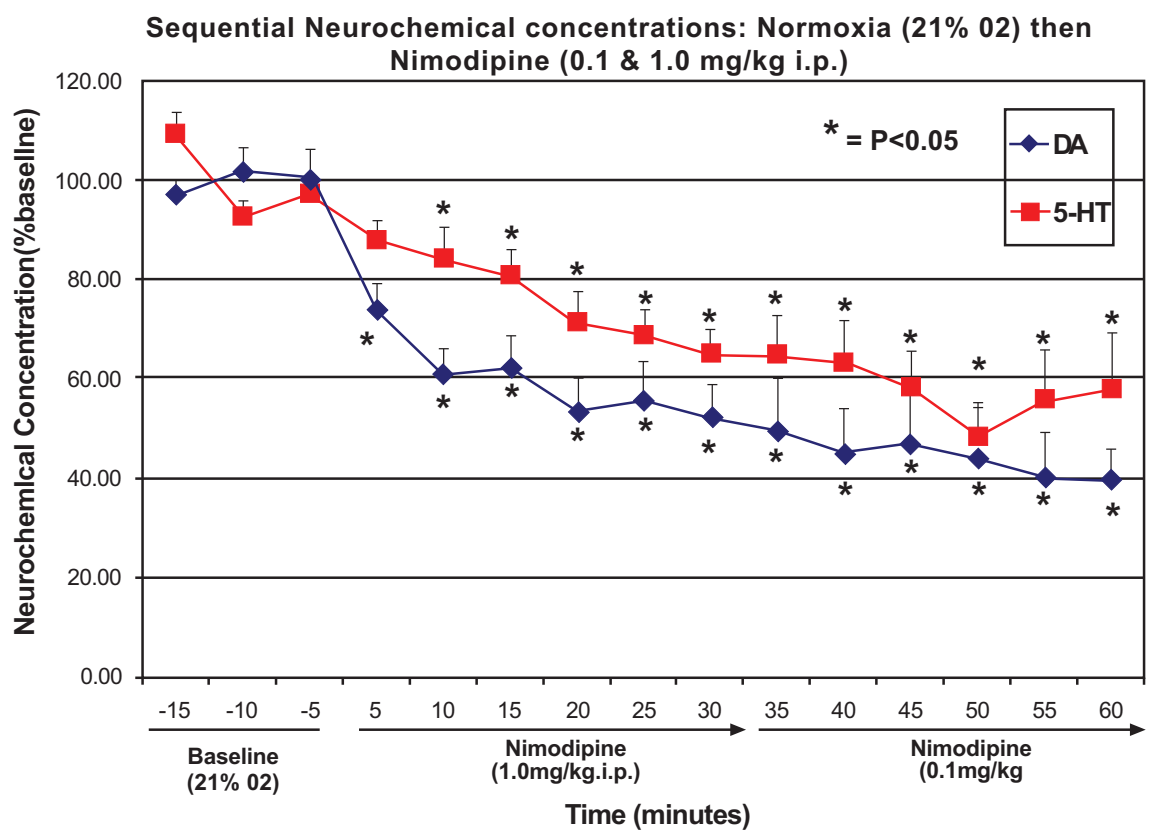

Fig. (4). Sequential Neurochemical concentrations: Normoxia $(21 \% \mathrm{O} 2)$ then Nimodipine $(0.1 \& 1.0 \mathrm{mg} / \mathrm{kg}$ i.p.). NMI signals for each neurochemical are expressed as percentages of baseline values (100\%) obtained over fifteen minutes. Treatment values were averaged over each sequential thirty minute period following each intervention. During normoxia, the injection of NIMO $(0.1 \mathrm{mg} / \mathrm{kg}) \mathrm{resulted}$ in the decrease of DA concentrations to $40 \%(\mathrm{SEM}=3$ ) and 5-HT to 23\% (SEM=4) below baseline. Under continuing normoxia, NIMO (1.0mg/kg) caused DA to fall to 55\% $(\mathrm{SEM}=2)$ and 5-HT to $42 \%(\mathrm{SEM}=2)$ below baseline. Measurements were analyzed with ANOVA with post hoc Tukeys test. P values less than 0.05 were considered significant.

and 5 -HT to $23 \%(\mathrm{SEM}=4)$ below baseline. Under continuing normoxia, NIMO $(1.0 \mathrm{mg} / \mathrm{kg})$ caused DA concentrations to fall to $55 \%(\mathrm{SEM}=2)$ and $5-\mathrm{HT}$ to $42 \%(\mathrm{SEM}=2)$ below baseline. NIMO treatment during normoxia caused the concentration of each neurochemical to fall below baseline. NIMO treatment did not significantly change SpO2 from baseline during the study period. Temperature remained stable throughout the study period at 37.1 degrees (SEM 0.1) Centigrade.

\section{DISCUSSION}

The present study reinforces our report that moderate hypoxia increases synaptic concentrations of DA and 5-HT in the dorsal striatum of Sprague-Dawley rats [9]. In that study $12.5 \% \mathrm{O} 2$ continually increased DA by $79 \%$ and 5 -HT by $26 \%$ above baseline over a period of 90 minutes. In addition, that study demonstrated that multiple episodes of mild to moderate hypoxia lead to prolonged elevations of NT despite the return of blood PO2 values to normal. Herein, NMI signals for DA and 5-HT showed continual increase during $10 \% \mathrm{O} 2$ over periods of up to 100 minutes. NIMO caused hypoxic and normoxic concentrations of each neurochemical to fall towards baseline and below baseline respectively. Another study showed that NIMO administered immediately after a $1 \mathrm{~h}$ exposure to $10 \% \mathrm{O} 2$ preserved short term working memory in mice [10]. These results taken together strengthen the original hypothesis that moderate hypoxia is linked to $\mathrm{Ca} 2+$ homeostasis, NT dysregulation, and ultimately cognitive dysfunction.

Moderate hypoxia may be linked to $\mathrm{Ca} 2+$ homeostasis and cognitive dysfunction in a number of ways. Cortical neurons have high metabolic demand, poor energy storage capability (Lust, W.D., 1985) and are the most vulnerable neu- rons to energy deprivation [16]. In the mammalian brain, 40$60 \%$ of ATP production at rest and more during activity maintains ion gradients [17]. Hypoxia decreases oxidative metabolism, causes membrane depolarization, and cellular ion homeostasis derangement. $\mathrm{Ca} 2+$ in neurons can increase by four orders of magnitude due to hypoxia $[17,18]$. In vitro studies of hypoxia and ischemia in rat cortex have shown that more than $85 \%$ of increased cytosolic $\mathrm{Ca} 2+$ was due to influx. $\mathrm{Ca} 2+$ inflow itself causes release of $\mathrm{Ca} 2+$ from internal stores [19, 20]. Increased cytosolic $\mathrm{Ca} 2+$ can lead to membrane damage, efflux of glutamate, decreased ATP synthesis, and lowered $\mathrm{pH}$ [15]. Diminished re-uptake of neurotransmitters contributes to an excitotoxic cascade [21].

Although the interaction of $\mathrm{N}$ and $\mathrm{P} / \mathrm{Q}$ type calcium channels with voltage-dependent and voltage-independent mechanisms is involved in the alteration of synaptic transmission [22], it is LTCC that are the link between membrane depolarization in neurons and cellular processes including synaptic efficacy and exocytosis in pathological states. In an in vitro model of rat pyramidal cortical neurons after energy failure most Ca2+ entered through LTCC [23] while in vitro studies of rat pheocromocytoma-12 cells showed that hypoxia caused Ca2+ influx through LTCC [24] . LTCC are present in neurons where they influence cellular functions involving neurotransmitters and the activation of other ion channels. Levels of intracellular $\mathrm{Ca} 2+$ affect multiple cellular functions, including the synthesis of several NT via $\mathrm{Ca} 2+/$ calmodulin-dependent protein kinase which activates tyrosine hydroxylase (the rate limiting step in the synthesis of DA) and tryptophan hydroxylase (the rate limiting step in the synthesis of 5-HT). LTCC blockers such as NIMO have significantly reduced membrane depolarization and internal $\mathrm{Ca} 2+$ levels in an in vitro model of oxygen 
O2/glucose deprivation using pyramidal cortical neurons [23]. Pharmacokinetic studies of NIMO show that after parental administration, effective brain levels can be achieved with doses as low as $0.25 \mathrm{mg} / \mathrm{kg}$ and at doses which minimize the risk of systemic hypotension [25].

Neurotransmitter function is very sensitive to hypoxia [26]. DA excess and acetylcholine (ACh) deficiency are considered to be both singly and in unison as parts of a final common pathway to delirium [27]. In an in vivo study of hypobaric hypoxia, NIMO increased rat hippocampal extracellular Ach [28]. Elevated concentrations of DA are intimately related to memory loss, delirium [27], and global neuronal oxidative stress [29]. DA metabolism is vulnerable to reactive oxygen species (ROS) while DA itself is a source of ROS [30]. Oxidative stress due to DA metabolism as well as a reduced capacity to respond to oxidative stress may explain some age-related cognitive deficits [31]. Both high and low concentrations of 5-HT are associated with delirium and are dependent upon availability of the amino acid LTryptophan which is the 5HT precursor [32] Thus, the maintenance of neurotransmitter concentrations within limits is important to the maintenance of cognitive function. The derangement and manipulation of neurotransmitter levels is a hallmark of the development and treatment of delirium. Levels of neurotransmitters, such as dopamine, serotonin, and glutamate are intimately involved with the formation of long term potentiation (LTP) which is considered to be the molecular basis of memory. In turn all are dependent on fine regulation of calcium homeostasis [8]. This study has shown that NIMO restores concentrations of DA and 5-HT in hypoxic rat striatum.

This study has a number of limitations. It is supportive of a role for moderate hypoxia upon LTCC and of the subsequent role of $\mathrm{Ca} 2+$ levels on cognitive function. However, no mechanistic link is demonstrated between moderate hypoxia and striatal NT concentrations. Further conclusions linking our studies are also problematic because while a great degree of commonality has been established between the physiology of rats and mice, further investigation is warranted to connect neurotransmitter concentrations to behavioral observations. In addition, the vascular effects of NIMO such as a possible increase in cerebral blood flow and a consequent increase in oxygen delivery cannot be eliminated within the bounds of this study. NIMO reduces deficits from cerebral hypoperfusion as well as ischemia because of its vasodilatory and direct neuronal actions [18]. However, NIMO has been shown to protect against ischemic neuronal damage without changing post ischemic cerebral blood flow [2]. Broderick and Gibson found that NT concentrations fell but remained elevated above baseline during intermittent hypoxic / normoxic periods and Haile et al. saw behavioral improvements with NIMO treatment during reperfusion periods after hypoxia. This study looked at NT concentrations during ongoing hypoxia but the presence of normoxic reperfusion or hypoxia during treatment is likely to be of consequence.

The vehicle for NIMO may be of concern in terms of NT levels but methanol is an alcohol that increases NT [33]. Polyethylene glycol is an emulsifier used with apomorphine for Parkinson's patients to assist in the increase of NT, particularly DA [34]. Thus, it is reasonable to conclude that
NIMO is producing the decrease in NT. Moreover, the sensitivity of the probe to the micron dimension of tissue can reflect fine differences in neuroanatomic substrates such as dorsal striatum while fine temporal resolution allows for consistent reading of NT concentrations [35]. The baseline values for each animal in this study are based on the control / normoxia values recorded for each subject before intervention confirming the same hypoxia results reported in [9]. The same animal control method allows for each animal to be studied as its own control and reveals consistent degrees of change between animals after intervention. The dorsal striatum was selected as the site for sensor placement because of its cholinergic input involved with cortico-basal ganglia information processing [36]. Sites more definitively associated with memory such as the hippocampus are studies in progress.

Nonetheless, we conclude that the present data may indicate a role for calcium mechanisms in hypoxia as mediated via NT. The data may well have implications for understanding and treating cognitive decline in the immediate postoperative period.

\section{ACKNOWLEDGEMENTS}

We would like to thank Dr. Sorosch Didehvar for his expert editing and Mr. Angel Martinez of New York University Medical School and Mr. Manuel Caballero of Bellevue Hospital Center for their expert technical assistance. We are also grateful for assistance from: The New York University Langone Medical Center Alzheimer's Disease Core Center for Grant AG08051-19, Medical Students Vivek Murthy, Helen Ho, and Jamil Shah of the Sophie Davis School of Biomedical Education at the City College of New York, The Leon Lowenstein Foundation, The F.M. Kirby Foundation and The Broderick Brain Foundation.

\section{REFERENCES}

[1] Micale V, Incognito T, Ignoto A, Rampello L, Spartà M, Drago F. Dopaminergic drugs may counteract behavioral and biochemical changes induced by models of brain injury. Eur Neuropsychopharmacol 2006; 16(3): 195-203.

[2] van der Mast RC. Serotonin and amino acids: partners in delirium pathophysiology? Sem Clin Neuropsychiat 2000; 5(2): 125-31.

[3] van der Mast RC. Postoperative delirium. Demen Geriat Cognit Disord 1999; 10: 401-5.

[4] Broderick PA. Studies of oxidative stress mechanisms using a morphine/ascorbate animal model and novel $\mathrm{N}$-stearoyl cerebroside and laurate sensors. J Neural Transmiss 2008; 115: 7-17.

[5] Bickler PE, Hansen BM. Causes of calcium accumulation in rat cortical brain slices during hypoxia and ischemia: role of ion channels and membrane damage. Brain Res 1994; 665(2): 269-76.

[6] Brown RC, Mark KS, Egleton RD, Davis TP. Protection against hypoxiainduced blood-brain barrier disruption: changes in intracellular calcium. Am J Physiol Cell Physiol 2004; 286(5): C1045-52.

[7] Silver IA, Erecinska M. Intracellular and extracellular changes of $[\mathrm{Ca} 2+]$ in hypoxia and ischemia in rat brain in vivo. J Gen Physiol 1990; 95: 837-66.

[8] Yamauchi T. Neuronal Ca2/Calmodulin-dependent protein Kinase II - discovery, progress in a quarter of a century, and perspective: Implication for Learning and Memory. Biologic Pharmaceutic Bull 2005; 28(8): 1342-54.

[9] Broderick PA, Gibson GE. Dopamine and serotonin in rat striatum during in vivo hypoxic-hypoxia. Metabol Brain Dis 1989; 4(2): $143-53$.

[10] Haile M, Limson F, Gingrich K, et al. Nimodipine prevents transient cognitive dysfunction after moderate hypoxia in adult mice. J Neurosurgic Anesthesiol 2009; 21(2): 140-4. 
[11] Davis JN, Carlsson A. The effect of hypoxia on monoamine synthesis, levels and metabolism in rat brain. J Neurochem 1973; 21(4): 783-90.

[12] Broderick PA, Pacia SV. Identification, diagnosis, and treatment of neuropathologies, neurotoxicities, tumors and brain and spinal cord injuries using microelectrodes with microvoltammetry. US. Patent \# 7 2006; 112: 319.

[13] Broderick PA, Ho H, Wat K, Murthy V.Biosensors Image Brain Neurotransmitters in vivo: Can an antihypertensive medication alter psychostimulant behavior? Sensors 2008; 8: 4033-61.

[14] Nunes JV, Broderick PA. Novel research translates to clinical cases of schizophrenic and cocaine psychosis. Neuropsychiat Dis and Treat 2007; 3(4): 475-85.

[15] Pellegrino LJ, Pellegrino AS, Cushman AJ. A stereotaxic atlas of the rat brain. Plenum Press, New York and London 1979.

[16] Siesjo BK. Historical overview: calcium, ischemia and death of brain cells. Annals of the New York Academy of Sciences 1988; 522: 638-61.

[17] Erecinska M, Silver IA. Tissue oxygen tension and brain sensitivity to hypoxia. Respir Physiol 2001; 128: 263-76.

[18] Yao H, Haddad GG. Calcium and pH homeostasis in neurons during hypoxia and ischemia. Cell Calcium 2004; 36(3-4): 247-55.

[19] Bickler PE, Donohoe PH. Adaptive responses of vertebrate neurons to hypoxia. J Exp Biol 2002; 205: 3579-86.

[20] Gröhn O, Kauppinen R. Intracellular chelation of calcium prevents cell damage following severe hypoxia in the rat cerebral cortex as studied by NMR spectroscopy ex vivo. Cell Calcium 1996; 20(6): $509-14$.

[21] Johnston MV. Cellular alterations associated with perinatal asphyxia. Clin Investig Med 1993; 16(2): 122-32.

[22] Elmslie KS. Neurotransmitter modulation of neuronal calcium channels. J Bioenerg Biomembr 2003; 35(6): 477-89.

[23] Pisani A, Calabresi P, Tozzi A, D'Angelo V, Bernardi G. L-Type $\mathrm{Ca} 2+$ Channel blockers attenuate electrical changes and $\mathrm{Ca} 2+$ rise induced by Oxygen/Glucose deprivation in cortical neurons. Stroke 1998; 29: 196-202.

[24] Premkumar DR, Adhikary G, Overholt JL, Simonson MS, Cherniack NS, Prabhakar NR. Intracellular pathways linking hypoxia to activation of c-fos and AP-1. Adv Exp Med Biol 2000; 475: 101-9.
[25] Heffez DS, Nowak TS, Passonneau JV. Nimodipine levels in the gerbil brain following parental drug administration. J Neurosurg 1985; 63(4): 589-92.

[26] van der Mast RC. Postoperative delirium. Dement Geriat Cognit Disord 1999; 10: 401-5.

[27] Trzepacz PT. Is there a final common neural pathway in delirium? Focus on acetylcholine and dopamine? Sem Clin Neuropsych 2000; 5(2): $132-48$

[28] Shukitt-Hale B, Stillman MJ, Levy A, Devine JA, Lieberman HR. Nimodipine prevents the in vivo decrease in hippocampal extracellular acetylcholine produced by hypobaric hypoxia. Brain Res 1993; 621(2): 291-5

[29] Micale V, Incognito T, Ignoto A, Rampello L, Spartà M, Drago F. Dopaminergic drugs may counteract behavioral and biochemical changes induced by models of brain injury. Eur Neuropsychopharmacol 2006; 16(3): 195-203

[30] Cantuti-Castelvetri I, Shukitt-Hale B, Joseph JA. Dopamine neurotoxicity: age dependent behavioral and histological effects. Neurobiol Aging 2003; 24(5): 697-706.

[31] Shukitt-Hale B, Erat SA, Joseph JA. Spatial learning and memory deficits induced by dopamine administration with decreased glutathione. Free Radic Biol Med 1998; 24(7-8): 1149-58.

[32] Lewis MC, Barnett SR. Postoperative delirium: the tryptophan dyregulation model. Med Hypotheses 2004; 63(3): 402-6.

[33] Randall S, Hannigan JH. In utero alcohol and postnatal methylphenidate: locomotion and dopamine receptors. Neurotoxicol Teratol 1999; 21(5): 587-93.

[34] Tsai MJ, Huang YB, Wu PC, et al. Oral apomorphine delivery from solid lipid nanoparticles with different monostearate emulsifiers: Pharmacokinetics and behavioral evaluations. J Pharm Sci 2010; [Epub ahead of print].

[35] Broderick PA. In vivo electrochemical studies of gradient effects of (SC) cocaine on dopamine and serotonin release in dorsal striatum of conscious rats. Pharmacol Biochem Behav 1993; 46: 973-84.

[36] Fino E, Glowinski J, Venance L. Effects of acute dopamine depletion on the electrophysiological properties of striatal neurons. Neurosci Res 2007; 58(3): 305-16.

(C) Haile et al.; Licensee Bentham Open.

This is an open access article licensed under the terms of the Creative Commons Attribution Non-Commercial License (http://creativecommons.org/licenses/by-nc/3.0/) which permits unrestricted, non-commercial use, distribution and reproduction in any medium, provided the work is properly cited. 